\section{Journal of Structural Biology}

www.elsevier.com/locate/yjsbi

Editorial

\title{
Special issue: Advances in molecular dynamics simulations
}

The past five years have seen an amazing boost in the field of biomolecular simulation. New approaches as well as dramatically improved computing power have made a wide panoply of molecular systems and processes accessible that can now be studied in detail by computer simulations. Large complexes such as the ribosome, molecular motors, or membrane-embedded pore complexes can now be simulated at the atomic level, with time scales up to microseconds being covered. We are on the cusp when the domains of biomolecular processes and biomolecular modeling merge.

Direct inspection of conformational motions and charge transfer reactions as the main determinants of protein function is currently becoming possible in an exponentially increasing number of cases, as is also evidenced by the similarly rapid increase of published simulation studies. On top of the more technical advances, new simulation methods have entered the stage, such as hybrid quantum mechanics/force field techniques (QM/MM), non-equilibrium simulations, enhanced sampling, coarse graining, and ensemble dynamics. These modern simulation tools have proven immensely successful in the endeavor to explain biological function from first principles.

Yet, as in many experimental fields, it is often difficult for the non-specialist to decide which questions can actually and reasonably be addressed by which simulation methodand which cannot. Even more difficult to assess for the non-expert is often the quality of a particular simulation, as well as the huge demand of computer power-i.e., of money-required.

This special issue titled Advances in Molecular Dynamics Simulations aims at alleviating these obstacles, which are in fact typical for a rapidly growing field. Addressing mainly the non-expert, the contributors provide a broad and application-oriented overview of leading-edge simulations studies on quite different biomolecular systems, employing, and thus illustrating, a similarly versatile toolbox of simulation techniques.

We start with very accurate and detailed-though expensive - quantum mechanics methods, move on to force field-based atomistic simulations of large systems and efficient sampling on long time scales, study the effect of external perturbations such as pressure, electric fields, and mechanical forces, and finish with highly efficient - though less accurate - coarse graining techniques.

While our selection is certainly - and necessarily-incomplete and guided by individual preferences, we do feel that the contributions collected within this special issue highlight a substantial fraction of what can be achieved today by atomistic computer simulations. Accordingly, we hope that this special issue of the Journal of Structural Biology will provide a point of reference for those active in the simulation field, and in particular that it will encourage colleagues to explore how simulations can complement and advance their experimental studies.

We thank the editors of the journal, Alasdair C. Steven and Wolfgang Baumeister, for their active support and advice, and the journal staff for their continuous and efficient help. We hope the reader will share our excitement and join us in tracking future advances in the area of biomolecular simulation that will open up new biological insights in the next five years to come.

Guest Editors

Helmut Grubmüller

Klaus Schulten 